\title{
LA OFERTA DE TURISMO RURAL EN EL NOROESTE DE LA REGIÓN DE MURCIA
}

\author{
Cayetano ESPEJO MARÍN \\ Departamento de Geografía Física, Humana y Análisis Regional \\ Universidad de Murcia
}

\begin{abstract}
Resumen: En los últimos años comienzan a desarrollarse en la comarca del Norneste de la Región de Murcia experiencias de promoción turística con carácter rural. En este artículo se analizan los productos turísticos que ofrece este espacio, el papel de la Administración en su desarrollo, el equipamiento turístico y las actuaciones previstas para el futuro.
\end{abstract}

Palabras clave: Administración, recursos naturales, tradiciones, turismo rural.

Abstract: Along the last years, new experiences of tourist promotion with a rural character have begun to be developed at the area north-west of Murcia. In this article we analyse the different tourist products which are offered by this area, as well as the paper realized by the administration for its development, the tourist resources and the actions foreseen for the future.

Key words: Administration, natural resources, traditions, rural tourism.

La oferta turística de la Región de Murcia se ha centrado tradicionalmente en el turismo de sol y playa, lo que ha dado lugar a un fuerte desarrollo urbanístico de los municipios del litoral.

Frente a este turismo tradicional del Mediterráneo español, en lo últimos años han empezado a desarrollarse experiencias de promoción turística en los municipios del interior de la Región, en concreto en la comarca del Noroeste murciano.

El resurgimiento del interés por todo lo relacionado con el medio ambiente y el contacto directo con el mismo ha contribuido a generar una nueva forma de utilización de los espacios rurales como áreas de esparcimiento que contrasta con las actividades realizadas tradicionalmente en estos espacios (MANERO MIGUEL, 1994). 
A esta situación se une la necesidad de buscar nuevas alternativas productivas a la agricultura de secanos tradicionales en crisis. El turismo está llamado a constituirse en un recurso económico de gran interés en este caso, dada la fácil adaptación de los recursos rurales en elementos de la oferta turística.

Concretamente nos referimos a lo que se ha dado en llamar agroturismo y turismo rural. Según ANDRÉS SARASA (1994), el primero se caracteriza como la "actividad de recepción y hospitalidad ejercidas por el empresario agrícola a través de la comercialización de la propia base territorial y como complemento de la actividad agraria", manteniéndose el aprovechamiento agrícola del suelo. El turismo rural se define "como la prestación de servicios turísticos demandados por motivos de vacaciones mediante el pago de los mismos y realizado en casas asentadas en el marco rural", en las que se obtiene alojamiento y servicios de restauración.

Algunas de las conclusiones a las que se llegan tras la celebración del I Encuentro Iberoamericano sobre Municipio y Turismo Rural, celebrado en Segovia en 1993 sirven para contribuir a definir y concretar la importancia del turismo rural. Entre ellas cabe señalar el papel social que puede desempeñar al proporcionar una actividad productiva a sectores de población que encuentran problemas para trabajar en la agricultura, como sucede especialmente en el caso de la mujer y de la juventud. Igualmente la aportación de riqueza a la sociedades agrarias en donde se desarrollan estas actividades, que por otra parte deben integrarse en el medio rural evitando su deterioro y potenciando sus valores tanto naturales como culturales.

Otro aspecto destacable de este tipo de actividad turística es que se dirige hacia una demanda de un poder adquisitivo medio-alto, que exige un disfrute de 1 a naturaleza contando con unas instalaciones adecuadas. Este turismo tiene la ventaja de que no es estacional, y que tampoco presenta problemas de saturación. Por otra parte puede ser un atractivo más para el turista que acude al litoral, al que se pueden ofertar rutas turísticas por el interior (VERA REBOLLO, 1991).

\section{EL TURISMO EN EL NOROESTE DE MURCIA}

La comarca del Noroeste de Murcia constituida por los municipios de Bullas, Calasparra, Caravaca de la Cruz, Cehegín y Moratalla es una de las que se ha incorporado a los proyectos de turismo rural. Se trata de una comarca deprimida económicamente, que se ha visto afectada por un continuo proceso de despoblamiento, dado que el desarrollo agrícola, entendiéndose por tal las denominadas nuevas agriculturas, no se ha producido, y la incidencia de los sectores industriales y de servicios no ha sido capaz de favorecer un crecimiento económico notable. 
Esta comarca ofrece unas características tanto naturales como históricoartísticas que la hacen especialmente adecuada para este tipo de turismo. Ofrece al visitante parajes naturales de gran interés: bosques mediterráneos en medio de una región árida, ríos y montañas, lugares donde practicar el senderismo, la caza, la pesca, y en definitiva para estar en contacto directo con la naturaleza.

La comarca del Noroeste ofrece la posibilidad de combinar las necesidades de ocio y descanso con el disfrute de tradiciones, paisajes, deporte, cultura, fiesta y actividades rurales.

La oferta deportiva de las tierras altas alterna la práctica de los deportes más convencionales con otros más atrevidos como: escalada, espeleología, parapente, a la delta, piragüismo, y carreras de orientación. Cabe citar, el descenso en cano por el Cañón de Almadenes en el río Segura, las rutas de espeleología en la Cueva del Puerto, la escalada de la Sierra del Molino, las rutas de senderismo en la zona alta de Moratalla, o los paseos a caballo por paisajes de una gran belleza.

El patrimonio histórico artístico es otro de los grandes atractivos de esta comarca. La riqueza histórica tiene su expresión en la diversidad de monumentos con los que cuenta. Además de yacimientos prehistóricos y de la Edad Antigua hay importantes conjuntos histórico-artísticos como los de Caravaca de la Cruz, Cehegín y Moratalla, además de fortalezas, edificios religiosos, escudos y casas señoriales. Las construcciones del siglo XVI en adelante se conservan bastantes, sobre todo las religiosas. Otro de los puntos de interés que se puede encontrar es la conservación de costumbres, oficios y labores tradicionales, entre los que destaca de un modo especial la artesanía del barro, del cáñamo, del esparto y de la madera.

Se pueden todavía ver las labores de la trilla en la era, como se ha hecho siempre, desmenuzando la parva con los clásicos trillos de pedernal tirados por caballerías y luego aventando ante la primera oportunidad de viento de poniente; los ancestrales ritos de la matanza del cerdo; los amasijos de pan cocido con leña de deshechos de enebro y carrasca; la elaboración de dulces artesanales hechos en el horno clásico árabe, y todo tipo de actividades agrícolas en los pequeños huertos familiares.

\section{EL PAPEL DE LA ADMINISTRACIÓN EN EL DESARROLLO TURISTICO}

Dado el carácter local de la mayor parte de las iniciativas de turismo rural resulta muy importante el papel que las administraciones locales deben desempeñar, 
sobre todo por los destacados efectos económicos que genera dicha actividad (SORET, 1994).

En lo que hace referencia a la comarca que estamos estudiando la actuación de la Administración Regional se centra en un doble objetivo: sacar a esta comarca de su estancamiento económico y diversificar la oferta turística regional para salvar una posible crisis del turismo de sol y playa. Se ha optado por un turismo calificado como interior, protegido y potenciado desde las Administraciones Autonómicas y Municipales.

La demanda del desarrollo de actividades turísticas y de ocio en espacios naturales hace imprescindible preservar los valores que constituyen el atractivo de dichos espacios impidiendo los impactos que acabarían con ellos. Por eso, es precisa una ordenación que regule este disfrute de la naturaleza y su conservación, y ahí es donde interviene la Administración, además de ordenar los recursos disponibles para esta actividad.

Los municipios afectados son los primeros que se preocupan por la regulación de estas actividades, acudiendo a experiencias de agroturismo y turismo rural que se han puesto en práctica en otros lugares. Se ha optado por la rehabilitación de casas en los cascos antiguos, evitando un excesivo impacto sobre la naturaleza y la intervención de las inmobiliarias.

Por su parte la Administración Autonómica, que cuenta con las competencias en materia turística desde 1982, se ha ocupado también de este aspecto. En lo que hace referencia a la Comarca del Noroeste, en 1992 se publica tn Decreto que regula la actividad de los alojamientos turísticos especiales en zonas de interior. En él quedan recogidas medidas que atienden a aspectos administrativos y fiscales, mientras que se presta escasa atención a la forma de ordenar y desarrollar un proyecto turístico que sirva para reactivar una zona económicamente deprimida sin que se vea afectada por los mismos impactos que han contribuido a la degradación del área litoral (ANDRÉS SARASA, 1994).

En 1989 se constituye una compañía mercantil anónima, Sociedad para la Promoción Turística del Noroeste S.A., integrada por el Ayuntamiento de Moratalla y el Instituto de Fomento de la Región de Murcia, con el objetivo de promover el desarrollo turístico del Noroeste, encargándose dicha sociedad de la gestión y promoción del turismo en dicha zona. Su actuación queda plasmada en la progresiva apertura del camping "La Puerta" (1989), el hotel "Cenajo" (1991) ambos en Moratalla y el camping "La Rafa" en Bullas (1992). En conjunto se trata de una oferta amplia, teniendo en cuenta que se parte de cero y diversificada, y que ha encontrado una buena respuesta en la demanda. 
A partir de 1993, con el fin de que cada municipio se encargue directamente de la gestión de sus recursos, se constituye la entidad "Región de Murcia Turística S.A." como central de reservas y organismo que coordina la gestiones municipales, encargándose también de la promoción.

Como complemento a los centros anteriores se crean los alojamientos rurales en casas antiguas restauradas con el apoyo de la Comunidad Europea y con el trabajo de los alumnos de las escuelas taller. Estas casas, tras cinco años de cesión a Región de Murcia Turística S.A. pasan de nuevo a su propietario, que puede optar por continuar con su explotación turística o dejarla para su uso privado.

Además de estas actividades el Instituto de Fomento suscribe en 1992 un convenio con el I.R.Y.D.A. para contribuir al desarrollo rural de los espacios deprimidos de la Región, a través de la iniciativa Leader, patrocinada por la Comunidad Europea. La Región de Murcia forma parte del programa Leader I mediante actuaciones en una zona que comprende las comarcas del Noroeste, Río Mula y las pedanías altas del municipio de Lorca. Dentro de las medidas a aplicar a los municipios afectados, la mayor parte de los proyectos corresponden al turismo rural.

\section{LA OFERTA DE EQUIPAMIENTOS TURÍSTICOS}

Tres son los tipos de alojamientos que se ofertan: las casas rurales, el Hotel Cenajo y los camping de La Puerta y La Rafa.

Como alojamientos rurales Leader se han rehabilitado molinos, casas de labranza, cortijos y casas en los cascos antiguos de los pueblos, totalmente restauradas con criterios y materiales de arquitectura popular, equipadas con cocina y baño completo, calefacción, agua caliente, ajuar y menaje completos, y todos los detalles necesarios para que la estancia del turista sea agradable.

En la actualidad la comarca del Noroeste cuenta con un total de 34 alojamientos rurales, distribuidos tal y como se puede ver en la Figura 1. Moratalla es el municipio que mayor número de casas y plazas ofrece, le sigue en importancia numérica en cuanto a viviendas Cehegín, a continuación Caravaca y Bullas. La capacidad de estos alojamientos queda expuesta en el cuadro I.

Además de las citadas casas se sitúan en la Comarca las denominadas hospederías rurales, viviendas que se alquilan por habitaciones. Su número asciende a 5 , tres de ellas se localizan en Moratalla, 1 en Cehegín y otra en Caravaca de la Cruz. 


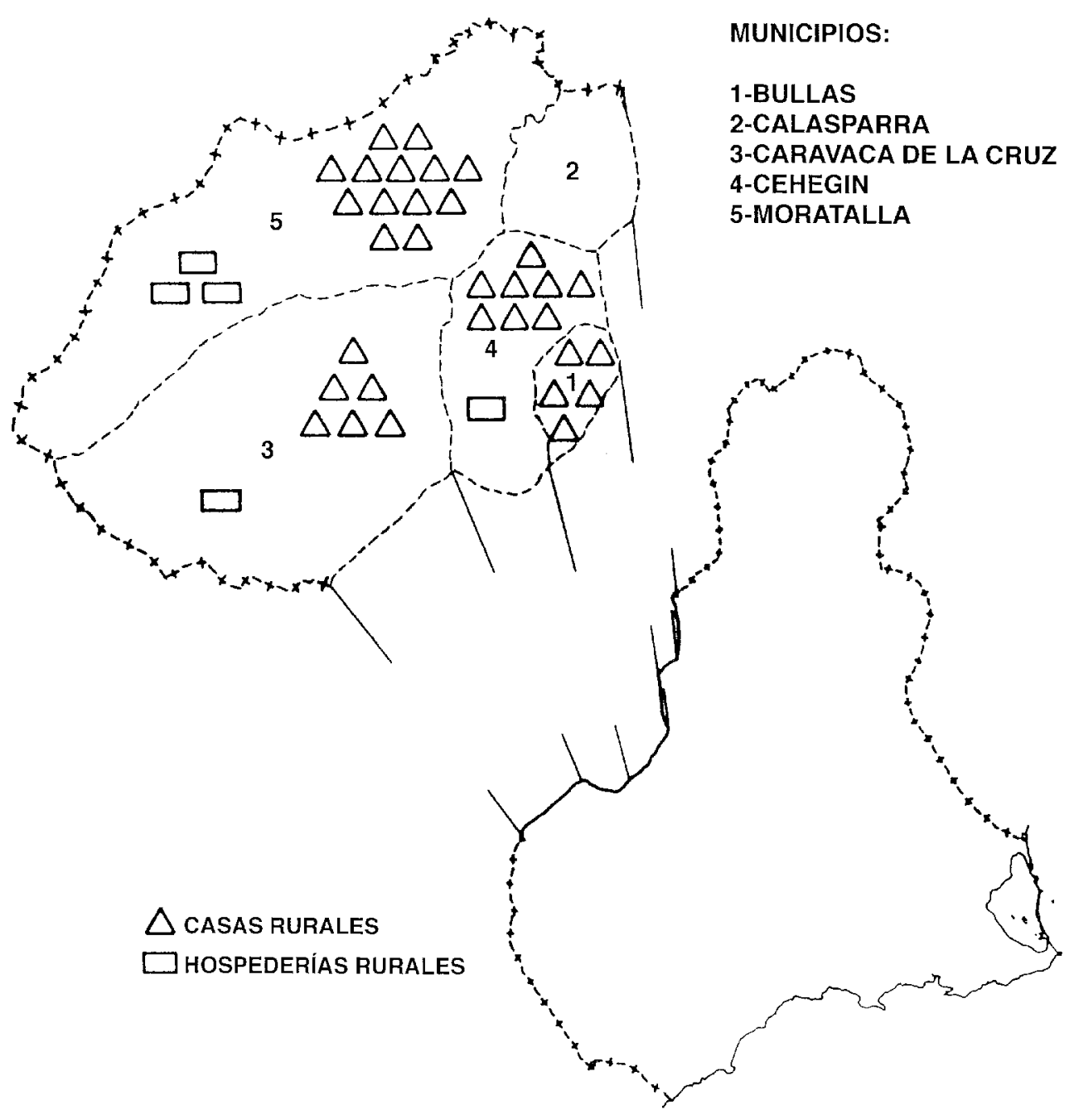

Figura 1.- Localización de los alojamientos rurales del Noroeste de Murcia. 
Con el fin de fomentar la ocupación de las casas rurales, así como conseguir que la estancia de los que las alquilan sea lo más agradable posible, la iniciativa Leader promueve el "Programa de Agroactividades", que ofrece a los visitantes que pernoctan en alojamientos rurales la posibilidad de participar activamente durante un día en una tarea tradicional, en convivencia con los habitantes de las pedanías y cortijadas, y acompañada por la gastronomía local.

Durante la temporada de otoño-invierno (1994-1995) se han programado los días del pan, de los dulces, de la matanza, del aguardiente, del queso, del aceite, de las esencias y de la miel, junto a las rutas artesanales: la tierra cocida de Valentín, pedanía de Moratalla y la ruta de los telares artesanos de Bullas y Mula, y dos rutas históricas: la de los castillos en Caravaca de la Cruz y Moratalla y la de las tierras altas entre El Sabinar y Benizar (Moratalla).

Un ejemplo de las actividades a desarrollar el día de las esencias: Se procede a la elaboración de las mismas mediante el llenado de la caldera con plantas aromáticas, se controla el fuego y el proceso de destilación, a continuación se procede a la decantación de la esencia y por último a la elaboración de linimento natural (alcohol de romero).

Estas primeras experiencias de animación turística en base a los oficios y rutas tradicionales y a la gastronomía están teniendo gran acogida entre los participantes.

En relación con estas actividades las iniciativas de reactivación promovidas por mujeres ya empiezan a ser notables. Hay que señalar que la participación de la mujer en todo lo relacionado con el turismo rural resulta bastante destacada. Esta actividad está generando un cierto volumen de empleo femenino bien en empresas familiares, o bien como asalariadas. Este hecho favorece la permanencia de la mujer en el medio rural, ofreciendo puestos de trabajo del sector servicios que son los que normalmente ocupan las mujeres (SABATÉ MARTINEZ, 1992).

El papel de la mujer en el turismo del Noroeste Murciano es ya destacable, sirva como ejemplo el hecho de que son propietarias de 11 de las 34 casas rurales, lo que significa que una de cada tres viviendas pertenece a ellas. En el caso de las hospederías rurales la participación es mayor, ya que de las cinco que hay en funcionamiento tres son de su propiedad, y en las otras dos su papel es muy importante.

El Hotel Cenajo se ubica en la antigua casa de administración de la obra del pantano del mismo nombre, dentro del término municipal de Moratalla, en el límite con la provincia de Albacete. Se trata de un hotel de 3 estrellas que cuenta con una capacidad de 130 plazas. En realidad, más que un hotel constituye un complejo con una singular oferta de ocio configurada por pistas de tenis, de squash, parque infantil, 
piscina climatizada, piscinas exteriores, sauna, paseos ciclistas y a caballo, y una diversidad de actividades organizadas por animadores turísticos. También cuenta con restaurante propio con cocina típica de la zona.

La puesta en marcha de estas instalaciones, que constituye una nota de calidad para el incipiente turismo del noroeste, fue complicada y llena de dificultades para adaptar unas antiguas instalaciones al complejo de ocio que hoy constituye. Las campañas publicitarias de promoción en base al entorno en que se ubica dieron una positiva respuesta. Campañas no sólo orientadas al ocio y descanso, sino que se introducen en la más reciente modalidad turística de las reuniones de empresas, para lo que está especialmente acondicionado, además de viajes de recompensa. Como ejemplo de esta tipología cabe señalar la realización de Cursos de Verano para estudiantes de la Universidad de Murcia (ANDRÉS SARASA, 1994).

El camping La Puerta en el municipio de Moratalla,es un camping de segunda categoría, situado en el paraje de La Puerta, a $8 \mathrm{~km}$. de Moratalla y a $20 \mathrm{Km}$. de Caravaca de la Cruz, con una capacidad para 600 personas y está abierto todo el año. Cuenta con restaurante, cafetería, supermercado, piscina, pista polideportiva, cabañas de madera, programa de animación semanal, barbacoas y fuentes de agua potable. También ofrece animación semanal con actividades de aerobic, senderismo, competiciones deportivas, juegos de piscina, fiestas infantiles, juegos de baile y música ambiental.

La zona de acampada se sitúa en un bosque de 20.000 metros cuadrados, y las parcelas cuentan con conexiones de electricidad, agua y televisión. También se ofrecen bungalows amueblados con 2 ó 3 dormitorios.

El camping La Rafa en el municipio de Bullas, situado en el paraje La Rafa, también de segunda categoría, con una capacidad para 350 personas, permanece abierto todo el año. La zona de acampada cuenta con 100 parcelas con conexiones de electricidad, agua, televisión y desagüe. Dispone de piscinas, solarium, pista polideportiva, tenis, cafetería, restaurante, supermercado, programas de animación, y parque infantil

La comarca del Noroeste cuenta también con otros locales en los que se pueden alojar y disfrutar de la gastronomía local, nos referimos a hoteles, hostales y restaurantes. Caravaca de la Cruz dispone de 105 plazas hoteleras en 4 establecimientos, Cehegín 57 plazas, Calasparra 45, y Bullas 8 plazas. Otro tipo de establecimientos existentes $y$ a tener en cuenta es el número de restaurantes, que asciende a 24 en Caravaca de la Cruz, 8 en Cehegín y Moratalla, y 7 en Bullas y Calasparra. 
Tabla 1.- Capacidad de los alojamientos rurales del Noroeste de Murcia. Fuente: Región de Murcia Turística. S.A.

\begin{tabular}{|c|c|c|c|c|c|c|c|c|c|c|}
\hline $\begin{array}{c}\text { Capacidad: } \\
\text { Plazas }\end{array}$ & \multicolumn{2}{|c|}{ BULLAS } & \multicolumn{2}{c|}{ CARAVACA } & \multicolumn{2}{c|}{ CEHEGIN } & \multicolumn{2}{c|}{ MORATALLA } & \multicolumn{2}{c|}{ TOTAL } \\
\cline { 2 - 11 } & Número & Plazas & Número & Plazas & Número & Plazas & Número & Plazas & Número & Plazas \\
\hline Menos de 4 & 1 & 2 & & & & & & & 1 & 2 \\
\hline 4 & 2 & 8 & 2 & 8 & 5 & 20 & 3 & 12 & 12 & 48 \\
\hline 5 & & & & & 2 & 10 & 1 & 5 & 3 & 15 \\
\hline 6 & 2 & 12 & 2 & 12 & 1 & 6 & 4 & 24 & 9 & 54 \\
\hline 7 & & & & & & & 2 & 14 & 2 & 14 \\
\hline 8 y más & & & 2 & 18 & & & 5 & 43 & 7 & 61 \\
\hline TOTAL & 5 & 22 & 6 & 38 & 8 & 36 & 15 & 98 & 34 & 194 \\
\hline
\end{tabular}

\section{ACTUACIONES FUTURAS}

El módulo de Desarrollo y Promoción de Cehegín está buscando la financiación necesaria para remodelar los edificios que jalonan la antigua línea férrea entre Baños de Mula y Caravaca de la Cruz, con la finalidad de reconvertirlos en hospederías, restaurantes o salas de exposiciones dentro de la puesta en marcha del denominado "pasillo verde" que unirá ambas poblaciones. Para la realización de estas actuaciones se podría recurrir tanto a los fondos comunitarios del programa Leader, como a particulares, y es intención de los responsables que los trabajos de remodelación se lleven a cabo por los alumnos de las distintas escuelas taller de la zona, tal y como ha ocurrido hasta ahora.

La línea verde entre Baños de Mula y Caravaca de la Cruz supone una inversión de 300 millones de pesetas, y estaba prevista su construcción para uso recreativo antes del año 1996, según convenio firmado por la Comunidad Autónoma de la Región de Murcia, la Fundación de Ferrocarriles Españoles y la Federación de Municipios de la Región de Murcia.

La finalidad de este proyecto es ampliar la oferta turística de la comarca del Noroeste. Este pasillo verde, con una longitud de $48 \mathrm{~km}$ permitirá la práctica, a pie o en bicicleta del denominado turismo ecológico. El recorrido sigue el valle del río Mula y las localidades de Mula, Bullas, Cehegín y Caravaca, se sitúa en un paisaje árido con abundantes cárcavas, así como zonas de pinares y almendros. 
A la oferta ecológica de este pasillo verde, primero en la Región, se sumaría también la oferta cultural con la posibilidad de visitar los cascos históricos de Mula, Cehegín y Caravaca de la Cruz, los restos arqueológicos de la ciudad de Begastri, así como el museo de arte ibérico de El Cigarralejo.

Debido a que en octubre de 1996 el proyecto para recuperar el trazado ferroviario como ruta turística está paralizado, se celebra en el Ayuntamiento de Caravaca un pleno extraordinario en el que con los votos a favor de los tres grupos políticos con representación municipal se acuerda reclamar la incorporación en los presupuestos generales del Estado del proyecto Vía verde por valor de 221 millones de pesetas, así como la contratación de las obras en el primer trimestre del próximo año. También se aprueba instar al Consejo de Gobierno de la Comunidad Autónoma para que se incluyan partidas en los presupuestos regionales que posibiliten la puesta en marcha del citado proyecto.

El alcalde de Caravaca señala en el citado pleno que "este proyecto que se había incluido entre las prioridades del gobierno anterior, permitiría favorecer el desarrollo de nuestros municipios, ya que serviría para potenciar y ampliar la oferta medioambiental y turística que, acompañado con le resto de programas e iniciativas en favor del turismo rural, permitirán un desarrollo sostenido de nuestros pueblos. Es una pena que algo que venimos reclamando se haya parado por cuestión de los recortes presupuestarios y por las prioridades marcadas por los nuevos ministerios, a pesar de que el proyecto está redactado por la Fundación de Ferrocarriles Españoles; las diferentes administraciones implicadas en este proyecto debemos dar un paso hacia adelante y reclamar con firmeza la ejecución de la Vía Verde del Noroeste" (Diario "La Verdad" 16-Octubre-1996).

Recientemente se ha inaugurado en Caravaca una oficina de turismo con la finalidad de impulsar la promoción exterior de la comarca. Esto ha sido posible gracias a un convenio firmado entre el Ayuntamiento y la Comunidad Autónoma, que aportó dinero para la señalización informativa y turística así como para la promoción del Año Jubilar, recientemente clausurado (Diario "La Verdad" 8-Abril-1997).

\section{BIBLIOGRAFÍA}

ANDRÉS SARASA, J.L. (1994): "El turismo de interior en la Región de Murcia. Utopías y demagogias", Papeles de Geografía, 20, 107-119.

BACHILLER FERNÁNDEZ, J.M. (1994): "El turismo rural como propuesta de revitalización económica de áreas rurales desfavorecidas: el Suroeste Soriano", Ería, 33, 25-39. 
BARDÚN FERNÁNDEZ, E. (1987). "Turismo rural en España. Algunas iniciativas públicas", En Turismo en el medio rural, Oviedo, Consejería de Industria, Comercio y Turismo del Principado de Asturias, 9-26.

BARDÚN FERNÁNDEZ, E. (1990): "Consideraciones sobre el turismo rural en España y medidas de desarrollo", Revista de Estudios Turísticos, 108, 61-82.

BOTE GÓMEZ, V. (1988): Turismo en espacio rural. Rehabilitación del patrimonio sociocultural y de la economía local, Madrid, Ed. Popular, 134.

GALIANO, E. (1991): "El turismo rural en España", Revista de Estudios Turísticos, 110, 39-46.

MANERO MIGUEL, F. (1994): "Ordenación del territorio y turismo rural", en I Encuentro iberoamericano sobre Municipio y Turismo Rural, 135-153.

NAVALÓN GARCÍA, M.R. (1995): "El turismo rural como generador de rentas complementarias frente a la difusión del modelo turístico litoral en los municipios de la montaña prelitoral alicantina: Senija, Llíber, Jalón y Alcalalí", En La formació, la rehabilitació i les noves modalitats turistiques. III Jornades de Geografia del Turisme, Palma, Universitat de les Illes Balears, 235247.

SABATE MARTÍNEZ, A.(1992): "La participación de las mujeres en la reactivación de zonas rurales desfavorecidas", En Desarrollo local y medio ambiente en zonas desfavorecidas, Madrid, Ministerio de Obras Públicas y Transportes, 123-138.

SORET, P. (1994): "El papel de los entes locales en el desarrollo del turismo rural", En I Encuentro Iberoamericano sobre Municipio y Turismo Rural, 73-82.

VERA REBOLlo, J.F. (1991): "El turismo", En Atlas Región de Murcia, Murcia, La Opinión de Murcia, 301-312. 